\title{
Ecofriendly Synthesis of Anisotropic Gold Nanoparticles: A Potential Candidate of SERS Studies
}

\author{
Ujjwala Gaware, Vaishali Kamble, and Balaprasad Ankamwar \\ Bioinspired Materials Science Laboratory, Department of Chemistry, University of Pune, Ganeshkhind, Pune 411007, India
}

Correspondence should be addressed to Balaprasad Ankamwar, bankamwar@yahoo.com

Received 29 July 2012; Accepted 5 October 2012

Academic Editor: Ujjal Kumar Sur

Copyright ( $) 2012$ Ujjwala Gaware et al. This is an open access article distributed under the Creative Commons Attribution License, which permits unrestricted use, distribution, and reproduction in any medium, provided the original work is properly cited.

Ecofriendly synthesis of nanoparticles has been inspiring to nanotechnologists especially for biomedical applications. Moreover, anisotropic particle synthesis is an attractive option due to decreased symmetry of such particles often leads to new and unusual chemical and physical behaviour. This paper reports a single-step room-temperature synthesis of gold nanotriangles using a cheap bioresource of reducing and stabilizing agent Piper betle leaf extract. On treating aqueous chloroauric acid solution with Piper betle leaf extract, after $12 \mathrm{hr}$, complete reduction of the chloroaurate ions was observed leading to the formation of flat and single crystalline gold nanotriangles. These gold nanotriangles can be exploited in photonics, optical coating, optoelectronics, magnetism, catalysis, chemical sensing, and so forth, and are a potential candidate of SERS studies.

\section{Introduction}

The synthesis of metal and semiconductor nanoparticles has innumerable opportunities of research due to their present and future applications in biosensing [1], chemical sensing [2], recording media [3], optoelectronics [4], and catalysis [5]. Masatake has reported [6] gold as a novel catalyst in the 21st century: it's preparation, working mechanism, and application as the catalyst in $\mathrm{CO}$ oxidation. The majority of earlier research has focused on isotropic, that is, spherical particles. However, anisotropic particles are particularly interesting because the decreased symmetry of such particles often leads to new and unusual chemical and physical properties [7]. In this context, ecofriendly biosynthesis protocols are better roadmap to avoid adverse effects of nanomaterials especially in medical applications. Moreover, use of plant extracts as a reducing and capping agent for the synthesis of nanoparticles could be advantageous over other environmentally benign biological processes by eliminating the elaborate process of maintaining cell cultures. It can also be suitably scaled up for the large-scale synthesis of nanoparticles. The biosynthesis of platinum nanoparticles using Diospyros kaki leaf extract [8], silver nanoparticles using leaf extracts [9], silver and gold nanoparticles using phyllanthin [10], Clove extract [11], and within live Alfalfa plants in solid media [12] has been demonstrated. In recent studies, we have reported on the synthesis of gold nanoparticles by the reduction of aqueous $\mathrm{AuCl}_{4}{ }^{-}$ions using Cymbopogon flexuosus [13], Tamarindus indica [2], Emblica officinalis [14], Terminalia catappa [15], Murraya koenigii and Citrus limonum leaf extracts [16].

In the case of bioreduction of aqueous gold ions by lemongrass extract, we did observe the formation of a large percentage of single crystalline, highly (111)-oriented gold nanotriangles with interesting optical absorption in the near infrared region of the electromagnetic spectrum [13]. Preliminary studies on the lemongrass extract and the gold nanotriangles indicated that ketones/aldehydes present in the extract may play an important role in directing the shape evolution in these nanostructures [13]. In order to test whether this hypothesis is true, we have looked at the composition of other plants for possible presence of such molecules and have identified the Piper betle plant as a potential candidate for shape-controlled synthesis of gold nanoparticles. This paper elaborates below the reaction of aqueous chloroaurate ions with Piper betle leaf extract results 
in the formation of single crystalline sharp vertices and truncated flat gold nanotriangles in large percentage with little number of hexagons. The edge-length of the nanotriangles varies from 660 to $1000 \mathrm{~nm}$ as would be expected from the highly anisotropic nature of the nanotriangles; they exhibit large absorption in the near infrared region. Surfaceenhanced Raman spectroscopy (SERS) technique is generally used to enhance Raman signals by factors of $10^{4}-10^{6}$ [1719] for detecting the molecules at low concentrations and to acquire information of the surface of materials. Sabur et al. [20] demonstrated glycine detection limit as low as $10^{-12} \mathrm{M}$ using SERS intensity optimization by controlling the size and shape of faceted gold nanoparticles. Moreover, Krpetić et al. [21] demonstrated that gold nanoparticles of different core sizes play an important role in the design of functional nanoparticles for colorimetric and SERSbased sensing applications, allowing controlled nanoparticles assembly and tunable sensor response for trace detection of $\mathrm{Ni}$ (II) ions in an aqueous solution. The recent findings used to improve the gold nanoparticle-based SERS substrates with ultrahigh sensitivity for the detection of bacterial spores [22] and gold nanoparticle-coated biomaterial as SERS microprobes [23]. These reports suggest that the ecofriendly synthesized anisotropic gold nanoparticles could be a potential candidate of SERS studies. Presented below are details of the investigation.

\section{Materials and Methods}

2.1. Biosynthesis of Anisotropic Gold Nanoparticles. The broth used for the reduction of $\mathrm{Au}^{3+}$ ions to $\mathrm{Au}^{0}$ was prepared by taking $24 \mathrm{~g}$ of thoroughly washed and finely cut Piper betle leaves in a $500 \mathrm{~mL}$ Erlenmeyer flask with $100 \mathrm{~mL}$ of sterile distilled water. This mixture was then boiled for $5 \mathrm{~min}$ and filtered through four-fold muslin cloth on cooling to room temperature. In a typical experiment, $4 \mathrm{~mL}$ of this broth was added to $90 \mathrm{~mL}$ of $1 \times 10^{-3} \mathrm{M}$ aqueous chloroauric acid $\left(\mathrm{HAuCl}_{4}\right)$ solution at room temperature. The bioreduction of chloroaurate ions in solution was monitored by periodic sampling and measuring the UV-Vis-NIR spectra of the solutions.

2.2. UV-Vis-NIR Spectroscopy Studies. UV-vis-NIR spectroscopy measurements of the Piper betle leaves extractreduced gold nanotriangles were carried out as a function of time of reaction at room temperature on a JASCO dual-beam spectrophotometer (model V-570) operated at a resolution of $1 \mathrm{~nm}$.

2.3. X-Ray Diffraction (XRD) Measurement. X-ray diffraction measurement of gold nanotriangles powder was carried out on a Bruker axs (model D8 Advance) instrument operating at a voltage of $40 \mathrm{kV}$ and current of $40 \mathrm{~mA}$ with $\mathrm{Cu} \mathrm{K}_{\alpha}$ radiation.

2.4. Fourier Transform Infrared (FTIR) Spectroscopy Measurements. After complete reduction of $\mathrm{AuCl}_{4}{ }^{-}$ions by the Piper betle leaves extract, for the isolation of gold nanoparticles from the free proteins or other organic biomolecular compounds existing in the solution, the centrifugation was carried out at $6000 \mathrm{rpm}$ for $15 \mathrm{~min}$. Thus, obtained gold nanoparticle pellets after centrifugation were redispersed in water prior to FTIR analysis. Films of the purified gold nanoparticles were deposited on Si (111) wafers by simple drop coating and were subjected to FTIR analysis on a Perkin-Elmer FTIR Spectrum One spectrophotometer in the diffuse reflectance mode at a resolution of $4 \mathrm{~cm}^{-1}$.

2.5. Transmission Electron Microscopy (TEM) Measurements. TEM samples of the gold nanotriangles were prepared by placing a drop of the nanoparticle solution on carbon-coated copper grids and allowing the solvent to evaporate; TEM measurements were performed on a JEOL model 1200EX instrument operated at an accelerating voltage at $120 \mathrm{kV}$.

\section{Results and Discussion}

The kinetics of reduction of aqueous chloroaurate ions during reaction with the Piper betle leaves broth was followed by UV-vis-NIR spectroscopy. It is well known that gold nanoparticles exhibit various shades of colors depending on size and shapes of nanoparticles, which also supports the coffee color observed in this study, which arises due to excitation of surface plasmon resonance (SPR) in the gold nanoparticles [24]. Figure 1(a) shows the UV-vis-NIR spectra recorded from the aqueous chloroauric acid Piper betle leaves broth reaction medium as a function of time of reaction. It is observed that as the reaction proceeds, the gold SPR band at ca. $551 \mathrm{~nm}$ steadily increases in intensity. This band is indicative of the presence of spherical nanoparticles in solution. In addition to the peak at $551 \mathrm{~nm}$, a progressive increase in the absorption at longer wavelengths into the near-infrared (NIR) region of the electromagnetic spectrum is observed. The UV-vis-NIR spectra indicate that the peak in the long wavelength absorption is well into the NIR (Figure 1(a)). The spectrum of the biologically synthesized gold nanoparticles now clearly shows a peak centered at $1213 \mathrm{~nm}$, after $12 \mathrm{hrs}$ of the reaction. The long wavelength absorption both in solution could be either due to aggregation of spherical gold nanoparticles in solution $[1,13]$ or due to formation of anisotropic nanoparticles [25]. In our earlier work, we observed sintering of small spherical gold nanoparticles at room temperature to single crystalline gold nanotriangles, suggesting that the nanoparticle surface is liquid-like [13]. Figure 1(b) shows a TEM image recorded from the biologically synthesized gold nanoparticles at the end of the reaction with Piper betle leaves extract. The TEM image shows (Figure 1(b)) that the gold nanoparticles are predominantly triangular morphology. The biosynthesized nanotriangles consist of a mixture of triangles, truncated triangles, and hexagons. The truncation appears to be a common feature in such disk-like metal nanostructures and has been repeatedly observed in chemically prepared gold $[26,27]$ and silver nanotriangles $[28,29]$. It is not clear what the reasons are for the formation of truncated nanotriangles. An analysis of the nanoparticles indicated that the percentage of gold nanotriangles/hexagons in the as-prepared reaction medium was ca. $60 \%$ but could be enhanced to nearly 


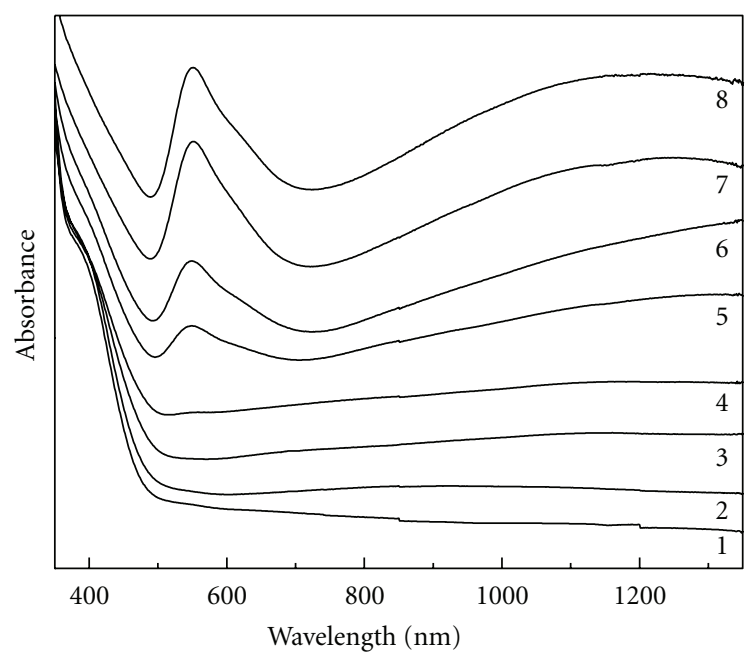

(a)

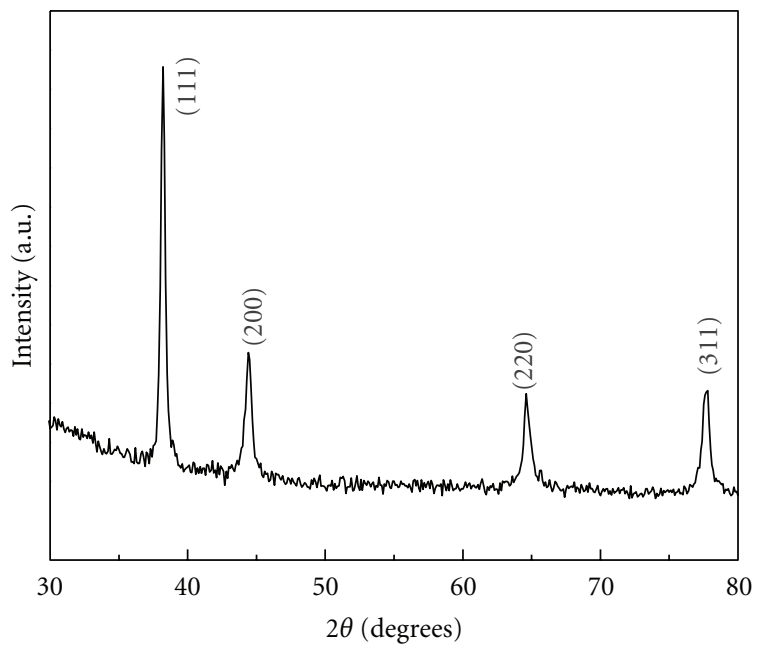

(c)

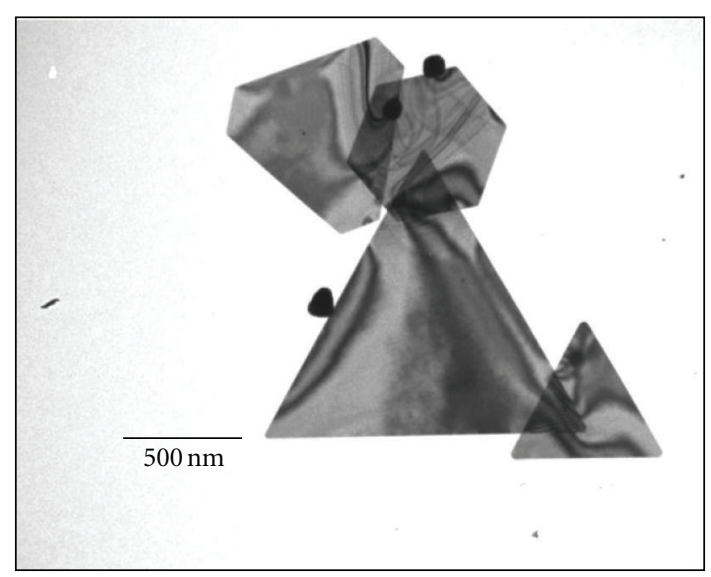

(b)

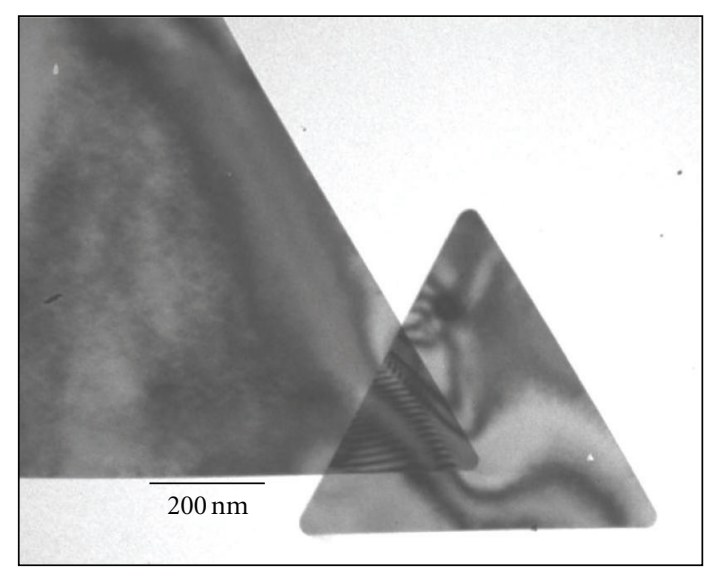

(d)

FIGURE 1: (a) UV-vis-NIR kinetics of the reaction of Piper betle leaf extract with aqueous chloroaurate ions. Curves 1-8 correspond to 0 , $10,60,120,180,240,480$, and 720 min after reaction, respectively. (b) Representative TEM micrographs of triangular gold nanoparticles obtained by the reduction of $\mathrm{AuCl}_{4}{ }^{-}$by Piper betle leaf extract. (c) XRD pattern of Piper betel leaf extract reduced nanotriangles with identified Bragg reflections. (d) A high magnified TEM micrograph of the single gold nanotriangles.

$90 \%$ through two cycles of centrifugation at $6,000 \mathrm{rpm}$, washing and redispersion. The gold nanotriangles in high magnified TEM image of Figure 1(d) shows considerable contrast along their surface. This contrast is due to strains in the nanoparticles indicating that they are extremely flat, thin, and easily buckle [30]. The TEM analysis thus clearly clarifies that the strong absorption in the NIR observed for the Piper betle leaf extract prepared gold nanoparticles is due to the formation of highly anisotropic nanostructures and not due to assembly of spherical gold nanoparticles. Here the edge-length of the nanotriangles varies from 660 to $1000 \mathrm{~nm}$ (Figure 1(b)); nanoprism structures with edge lengths as large as several micrometers have been synthesized earlier, but these have not exhibited the optical or chemical properties associated with their smaller analogs [31-33]. Technically, triangular nanoprisms contain three sharp vertices that contribute significantly to their optical and electronic properties [31,33]. In some cases, nanoprisms dimensions can be controlled in situ by adjusting experimental parameters, including metal ion and reducing agent ratios [34]. Earlier report has shown a mixture of spherical and polycrystalline nanoplatelet shapes with a low yield of either spherical or nanoplatelets [35]. In this paper bio-source of reducing and capping agent Piper betle leaves extract has resulted into high yield of formation of higher edge-length of single crystalline nanoprisms $(660-1000 \mathrm{~nm})$ compare to our earlier reports of gold nanoprisms synthesis using extracts of biosources Cymbopogon flexuosus (200-500 nm) [13] and Tamarindus indica $(100-500 \mathrm{~nm})$ [2].

Figure $1(\mathrm{c})$ shows the X-ray diffraction patterns of the gold nanotriangles obtained in the Piper betle leaves extract reaction. The $2 \theta$ values of the standard Au nanoparticles 


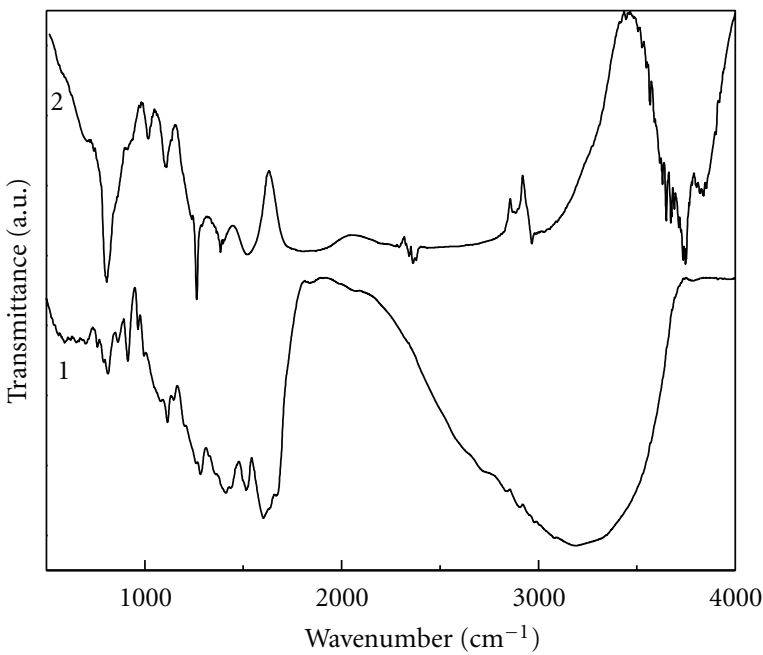

(a)

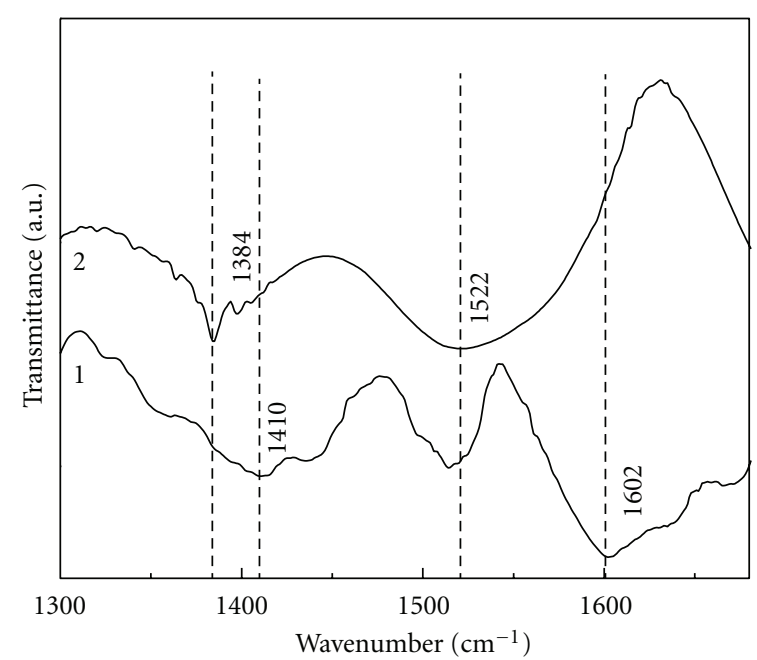

(b)
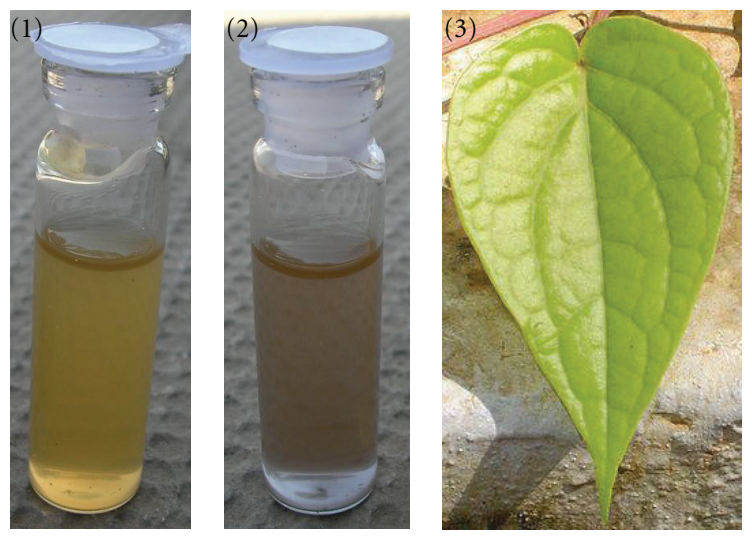

(c)

Figure 2: FTIR spectra of (1) pure Piper betle leaf broth and (2) Piper betle leaf-reduced gold nanoparticles in the wavenumbers range from (a) 400 to $4000 \mathrm{~cm}^{-1}$ and (b) 1300 to $1750 \mathrm{~cm}^{-1}$; (c) it shows aqueous extract of Piper betle leaf (1), Piper betle leaf-reduced gold nanoparticles (2), and Piper betle leaf (3).

$38.184,44.392,64.576$, and 77.547 degrees correspond to the Bragg reflections (111), (200), (220), and (311). Where as the $2 \theta$ values of the obtained gold nanotriangles $38.2,44.4$, 64.6 , and 77.8 degrees correspond to the Bragg reflections (111), (200), (220), and (311) that may be indexed on the basis of the fcc structure of gold. In consideration of wavelength of light source $(\lambda=1.54056 \AA)$, our $2 \theta$ values are almost matching with the JCPDF file no 04-0784 for gold. The (200), (220), and (311) Bragg reflections are extremely weak and considerably broadened relative to the intense (111) reflection. This interesting feature indicates that gold nanocrystals are highly anisotropic in nature and that the particles in the film are (111)-oriented.

FTIR measurements were carried out to identify the potential biomolecules in the Piper betle leaf broth responsible for the reduction of the chloroaurate ions and also the capping reagent responsible for the stability of the bioreduced gold nanoparticles. Bombay Piper betle leaves contain [36] reducing sugars (as glucose) 1.4-3.2\%, nonreducing sugars (as sucrose) $0.6-2.5 \%$, total sugars 2.4-5.6,
Starch 1.0-1.2\%, essential oil 0.8-1.8, and tannin 1.0-1.3. This composition data was used as a guideline to identify possible reducing and stabilizing biomolecules from the Piper betle leaves. Curves 1 and 2 of Figure 2(a) represent the FTIR spectrum of the Piper betle leaves extract and Piper betle leaves extract reduced gold nanoparticles with absorption bands at $759,792,812,864,914,965,996,1115,1147,1282$, $1410,1514,1602$, and $3195 \mathrm{~cm}^{-1}$ and $806,912,1018,1110$, $1263,1384,1522$, and $2966 \mathrm{~cm}^{-1}$, respectively.

The shoulder at $1602 \mathrm{~cm}^{-1}$, is characteristic of carbonyl stretch vibrations in ketones, aldehydes, and carboxylic acids. The $1616 \mathrm{~cm}^{-1}$ band is assigned to aromatic CC skeletal vibrations/ $\mathrm{N}-\mathrm{H}$ deformations, most likely from indoleacetic acid [13]. Curve 2 shows the FTIR spectrum of the Piper betle leaves extract reduced gold with the absorption bands at $1602 \mathrm{~cm}^{-1}$ and $1410 \mathrm{~cm}^{-1}$. The shift of the $1602 \mathrm{~cm}^{-1}$ band to $1522 \mathrm{~cm}^{-1}$ is attributed to binding of aldehydes/ketones with the gold nanoparticle surface [13]. The band at $1602 \mathrm{~cm}^{-1}$ is characteristics of carbonyl stretch vibrations [37], possibly from the acid groups present in 
the Piper betle leaf extract. The shift in the carbonyl stretch frequency $\left(1602 \mathrm{~cm}^{-1}\right)$ to lower wave numbers $\left(1522 \mathrm{~cm}^{-1}\right)$ followed by the disappearance of the $1602 \mathrm{~cm}^{-1}$ resonance may be due to its binding with the gold nanoparticle surface. A comparison of the two spectra also reveals the presence of prominent feature at ca. $3195 \mathrm{~cm}^{-1}$ in curve 1 while the $3195 \mathrm{~cm}^{-1}$ feature shifts to $2966 \mathrm{~cm}^{-1}$ due to coordination of the amine molecule with gold nanoparticles surface [38].

In our earlier report, we had mentioned that we believe the formation of gold nanotriangles is due to reduction of aqueous $\mathrm{AuCl}_{4}{ }^{-}$ions by the reducing sugars, Raveendran et al. [39] used the reducing sugar $\beta$-D-glucose as the reducing agent in the synthesis of green synthesis of silver nanoparticles. Bombay Piper betle leaves used in this paper contain reducing sugars (as glucose) 1.4-3.2\% [36]. FTIR also supports the aldehydes/ketones bind to the nascent spherical nanoparticles rendering them "liquid-like" and amenable to sintering at room temperature as we earlier reported [13]. We did control experiments with different amounts of Piper betle leaf extract keeping constant volume and concentration of chorloauric acid; in this study, we observed that slow reduction rate was favoring the formation of anisotropic structures such as triangular structure. This could be achieved by maintaining appropriate concentration ratio of precursor and leaf extract. This study helps to elucidate the role of economical bio-resource, that is, aqueous plant extracts in shape-directing factors involved in ecofriendly syntheses of anisotropic metallic nanoparticles. Moreover, these anisotropic metal nanoparticles show "lightning-rod effect," another kind of field enhancement refers to enhanced charge density localization at a tip or vertex of nanoparticles. When an electromagnetic field (e.g., laser light) excites the free electrons of a metallic tip, a highly localized, strong electric field develops at these sharp tips or vertex with large curvatures, leading to a large field enhancement in those regions. This is the reason for the high Surface Enhanced Raman Scattering (SERS) activity of anisotropic nanoparticles. In our laboratory studies on the use of synthesized anisotropic gold nanoparticles as an effective SERS active substrate are going on which will be communicated later. This biogenic synthesis of metal nanoparticles is also important in bionanotechnology and can be utilized as a light energy conversion devices by combine with an organic dyad.

\section{Conclusions}

The one step synthesis of stable gold nanotriangles in high concentration using Piper betle leaves extract has been demonstrated. The reducing sugars (as glucose) are mainly responsible for formation of metallic gold nanoparticles. The high absorption coefficient of these gold triangles in the NIR region can be exploited in fabricating photonic devices such as optical sensors and in hyperthermia of tumors [40]. Gold nanotriangles have also the characteristics required for chemical sensor development [2]. In addition to chemical sensing the work of bio-sensing, SERS studies and catalysis are also the best candidatures for these anisotropic gold nanoparticles. Moreover, these structures are especially interesting because they have plasmonic features in the visible and IR regions, can be prepared in high yield and readily functionalized with a variety of sulfur-containing adsorbates [41-43], and is currently being pursued.

\section{Acknowledgment}

The authors wish to express their appreciation to BCUD (BCUD/OSD/390; dated 16/11/2010), University of Pune for financial support.

\section{References}

[1] C. A. Mirkin, R. L. Letsinger, R. C. Mucic, and J. J. Storhoff, "A DNA-based method for rationally assembling nanoparticles into macroscopic materials," Nature, vol. 382, no. 6592, pp. 607-609, 1996.

[2] B. Ankamwar, M. Chaudhary, and M. Sastry, "Gold nanotriangles biologically synthesized using tamarind leaf extract and potential application in vapor sensing," Synthesis and Reactivity in Inorganic, Metal-Organic, and Nano-Metal Chemistry, vol. 35, no. 1, pp. 19-26, 2005.

[3] S. Sun, C. B. Murray, D. Weller, L. Folks, and A. Moser, "Monodisperse FePt nanoparticles and ferromagnetic FePt nanocrystal superlattices," Science, vol. 287, no. 5460, pp. 1989-1992, 2000.

[4] D. H. Gracias, J. Tien, T. L. Breen, C. Hsu, and G. M. Whitesides, "Forming electrical networks in three dimensions by self-assembly," Science, vol. 289, no. 5482, pp. 1170-1172, 2000.

[5] M. Valden, X. Lai, and D. W. Goodman, "Onset of catalytic activity of gold clusters on titania with the appearance of nonmetallic properties," Science, vol. 281, no. 5383, pp. 16471650, 1998.

[6] H. Masatake, "Gold as a novel catalyst in the 21st century: Preparation, working mechanism and applications," Gold Bulletin, vol. 37, no. 1-2, pp. 27-36, 2004.

[7] J. E. Millstone, S. J. Hurst, G. S. Métraux, J. I. Cutler, and C. A. Mirkin, "Colloidal gold and silver triangular nanoprisms," Small, vol. 5, no. 6, pp. 646-664, 2009.

[8] J. Y. Song, E. Y. Kwon, and B. S. Kim, "Biological synthesis of platinum nanoparticles using Diopyros kaki leaf extractBioprocess and Biosystems Engineering," vol. 33, pp. 159-164, 2010.

[9] J. Y. Song and B. S. Kim, "Rapid biological synthesis of silver nanoparticles using plant leaf extracts," Bioprocess and Biosystems Engineering, vol. 32, pp. 79-84, 2009.

[10] J. Kasthuri, K. Kathiravan, and N. Rajendiran, "Phyllanthinassisted biosynthesis of silver and gold nanoparticles: a novel biological approach," Journal of Nanoparticle Research, vol. 11, no. 5, pp. 1075-1085, 2009.

[11] A. K. Singh, M. Talat, D. P. Singh, and O. N. Srivastava, "Biosynthesis of gold and silver nanoparticles by natural precursor clove and their functionalization with amine group," Journal of Nanoparticle Research, vol. 12, no. 5, pp. 1667-1675, 2010.

[12] J. L. Gardea-Torresdey, E. Gomez, J. R. Peralta-Videa, J. G. Parsons, H. Troiani, and M. Jose-Yacaman, "Alfalfa sprouts: a natural source for the synthesis of silver nanoparticles," Langmuir, vol. 19, no. 4, pp. 1357-1361, 2003.

[13] S. S. Shankar, A. Rai, B. Ankamwar, A. Singh, A. Ahmad, and M. Sastry, "Biological synthesis of triangular gold nanoprisms," Nature Materials, vol. 3, no. 7, pp. 482-488, 2004. 
[14] B. Ankamwar, C. Damle, A. Ahmad, and M. Sastry, "Biosynthesis of gold and silver nanoparticles using Emblica Officinalis fruit extract, their phase transfer and transmetallation in an organic solution," Journal of Nanoscience and Nanotechnology, vol. 5, no. 10, pp. 1665-1671, 2005.

[15] B. Ankamwar, "Biosynthesis of gold nanoparticles (GreenGold) using leaf extract of Terminalia Catappa," E-Journal of Chemistry, vol. 7, no. 4, pp. 1334-1339, 2010.

[16] B. Ankamwar, Biosynthesis: An Eco-Friendly Approach of Nanomaterials Synthesis, Chemical and Biomedical Applications, VDM, 2010.

[17] P. Hildebrandt and M. Stockburger, "Surface-enhanced resonance Raman spectroscopy of Rhodamine 6G adsorbed on colloidal silver," The Journal of Physical Chemistry B, vol. 88, no. 24, pp. 5935-5944, 1984.

[18] X. M. Dou, Y. M. Jung, Z. Q. Cao, and Y. Ozaki, "Surfaceenhanced raman scattering of biological molecules on metal colloid II: effects of aggregation of gold colloid and comparison of effects of ph of glycine solutions between gold and silver colloids," Applied Spectroscopy, vol. 53, no. 11, pp. 1440-1447, 1999.

[19] H. X. Xu, J. Aizpurua, M. Kall, and P. Apell, "Electromagnetic contributions to single-molecule sensitivity in surfaceenhanced Raman scattering," Physical Review E, vol. 62, pp. 4318-4324, 2000.

[20] A. Sabur, M. Havel, and Y. Gogotsi, "SERS intensity optimization by controlling the size and shape of faceted gold nanoparticles," Journal of Raman Spectroscopy, vol. 39, no. 1, pp. 61-67, 2008.

[21] Ž. Krpetić, L. Guerrini, I. A. Larmour, J. Reglinski, K. Faulds, and D. Graham, "Importance of nanoparticle size in colorimetric and sers-based multimodal trace detection of $\mathrm{Ni}$ (II) Ions with functional gold nanoparticles," Small, vol. 8, pp. 707-714, 2012.

[22] H. W. Cheng and R. Q. Yu, "Nanoparticle-based substrates for surface-enhanced Raman scattering detection of bacterial spores," Analyst, vol. 137, pp. 3601-3608, 2012.

[23] G. V. Pavankumar, "Gold nanoparticle-coated biomaterial as SERS micro-probes," Bulletin of Materials Science, vol. 34, no. 3, pp. 417-422, 2011.

[24] P. Mulvaney, "Surface plasmon spectroscopy of nanosized metal particles," Langmuir, vol. 12, no. 3, pp. 788-800, 1996.

[25] E. Hao, K. L. Kelly, J. T. Hupp, and G. C. Schatz, "Synthesis of silver nanodisks using polystyrene mesospheres as templates," Journal of the American Chemical Society, vol. 124, no. 51, pp. 15182-15183, 2002.

[26] N. Malikova, I. Pastoriza-Santos, M. Schierhorn, N. A. Kotov, and L. M. Liz-Marzán, "Layer-by-layer assembled mixed spherical and planar gold nanoparticles: control of interparticle interactions," Langmuir, vol. 18, no. 9, pp. 36943697, 2002.

[27] Y. Shao, Y. Jin, and S. Dong, "Synthesis of gold nanoplates by aspartate reduction of gold chloride," Chemical Communications, vol. 10, no. 9, pp. 1104-1105, 2004.

[28] S. Chen and D. L. Carroll, "Synthesis and characterization of truncated triangular silver nanoplates," Nano Letters, vol. 2, no. 9, pp. 1003-1007, 2002.

[29] R. Jin, Y. Cao, C. A. Mirkin, K. L. Kelly, G. C. Schatz, and J. G. Zheng, "Photoinduced conversion of silver nanospheres to nanoprisms," Science, vol. 294, no. 5548, pp. 1901-1903, 2001.

[30] W. T. S. Huck, N. Bowden, P. Onck, T. Pardoen, J. W. Hutchinson, and G. M. Whitesides, "Ordering of spontaneously formed buckles on planar surfaces," Langmuir, vol. 16, no. 7, pp. 3497-3501, 2000.
[31] K. L. Kelly, E. Coronado, L. L. Zhao, and G. C. Schatz, "The optical properties of metal nanoparticles: the influence of size, shape, and dielectric environment," The Journal of Physical Chemistry B, vol. 107, no. 3, pp. 668-677, 2003.

[32] Y. L. Luo, "Large-scale preparation of single-crystalline gold nanoplates," Materials Letters, vol. 61, no. 6, pp. 1346-1349, 2007.

[33] K. L. Shuford, M. A. Ratner, and G. C. Schatz, "Multipolar excitation in triangular nanoprisms," The Journal of Chemical Physics, vol. 123, no. 11, pp. 114713-114722, 2005.

[34] G. S. Métraux and C. A. Mirkin, "Rapid thermal synthesis of silver nanoprisms with chemically tailorable thickness," Advanced Materials, vol. 17, no. 4, pp. 412-415, 2005.

[35] K. Sneha, M. Sathishkumar, S. Kim, and Y. S. Yun, "Counter ions and temperature incorporated tailoring of biogenic gold nanoparticles," Process Biochemistry, vol. 45, no. 9, pp. 14501458, 2010.

[36] A. Krishnamurthi, Ed., The Wealth of India, A Dictionary of Indian Raw Materials \& Industrial Products, vol. 8 of Raw Materials, National Institute of Science Communication, CSIR, New Delhi, India, 1998.

[37] V. Patil, R. B. Malvankar, and M. Sastry, "Role of particle size in individual and competitive diffusion of carboxylic acid derivatized colloidal gold particles in thermally evaporated fatty amine films," Langmuir, vol. 15, no. 23, pp. 8197-8206, 1999.

[38] D. V. Leff, L. Brandt, and J. R. Heath, "Synthesis and characterization of hydrophobic, organically-soluble gold nanocrystals functionalized with primary amines," Langmuir, vol. 12, no. 20, pp. 4723-4730, 1996.

[39] P. Raveendran, J. Fu, and S. L. Wallen, "Completely "green" synthesis and stabilization of metal nanoparticles," Journal of the American Chemical Society, vol. 125, no. 46, pp. 1394013941, 2003.

[40] L. R. Hirsch, R. J. Stafford, J. A. Bankson et al., "Nanoshellmediated near-infrared thermal therapy of tumors under magnetic resonance guidance," Proceedings of the National Academy of Sciences of the United States of America, vol. 100, no. 23, pp. 13549-13554, 2003.

[41] J. E. Millstone, S. Park, K. L. Shuford, L. Qin, G. C. Schatz, and C. A. Mirkin, "Observation of a quadrupole plasmon mode for a colloidal solution of gold nanoprisms," Journal of the American Chemical Society, vol. 127, no. 15, pp. 5312-5313, 2005.

[42] C. Xue and C. A. Mirkin, "pH-switchable silver nanoprism growth pathways," Angewandte Chemie International Edition, vol. 46, no. 12, pp. 2036-2038, 2007.

[43] R. Jin, Y. C. Cao, E. Hao, G. S. Métraux, G. C. Schatz, and C. A. Mirkin, "Controlling anisotropic nanoparticle growth through plasmon excitation," Nature, vol. 425, no. 6957, pp. 487-490, 2003. 


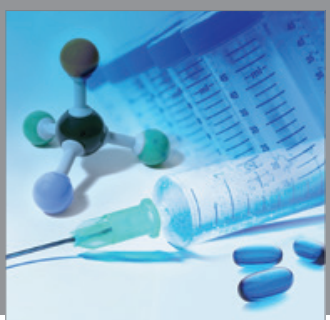

International Journal of

Medicinal Chemistry

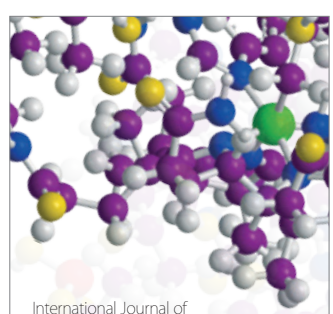

Carbohydrate Chemistry

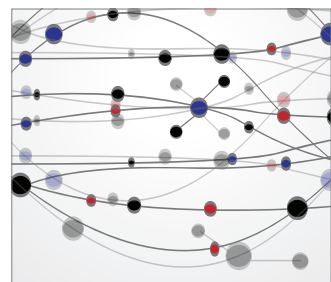

The Scientific World Journal
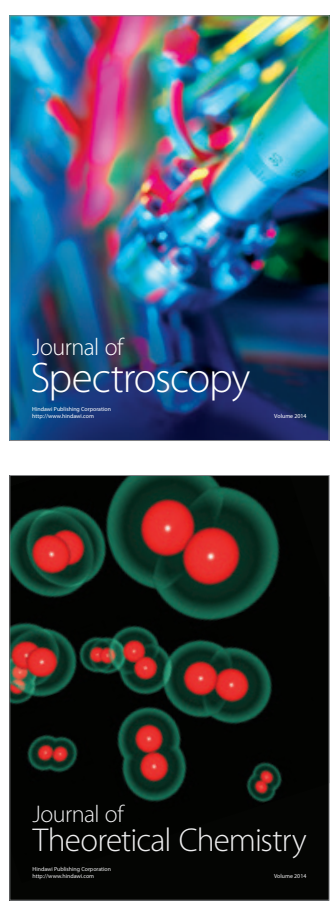
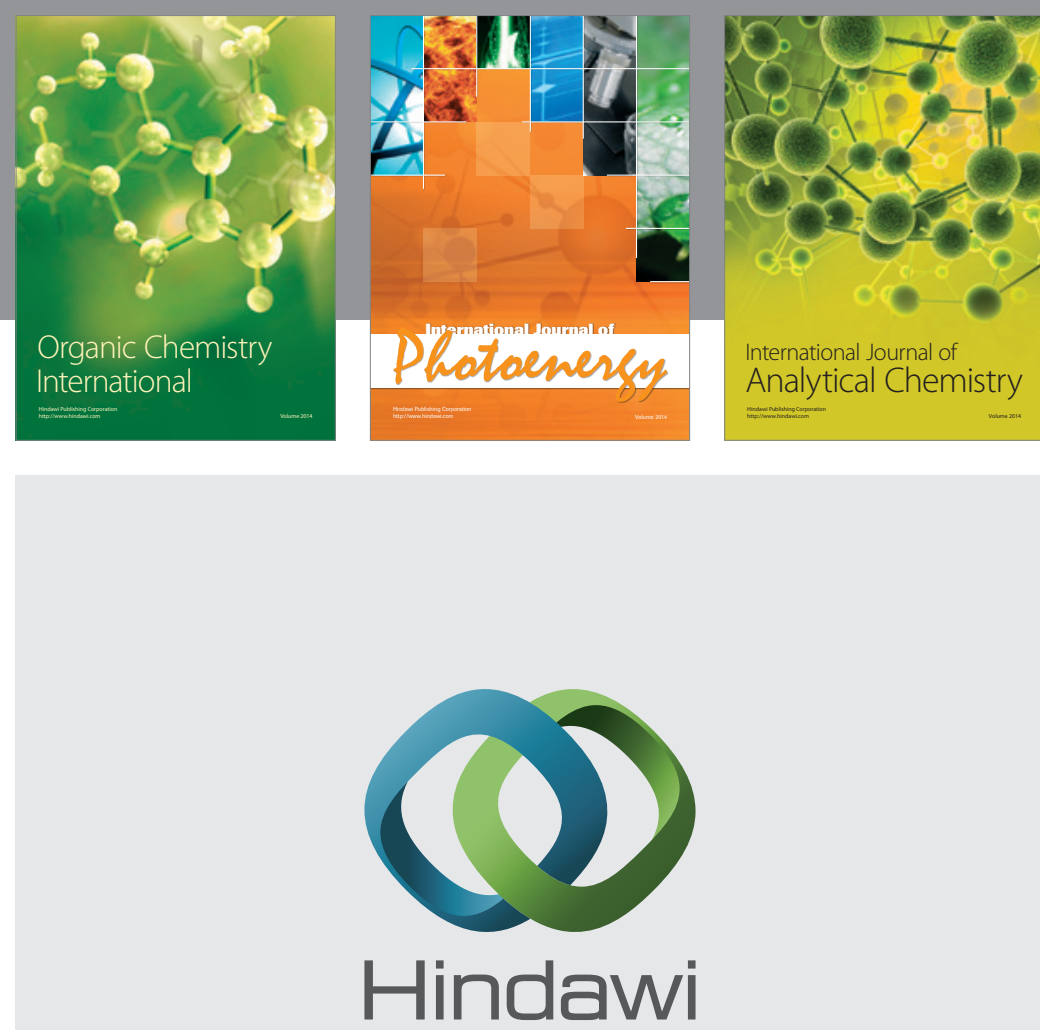

Submit your manuscripts at

http://www.hindawi.com
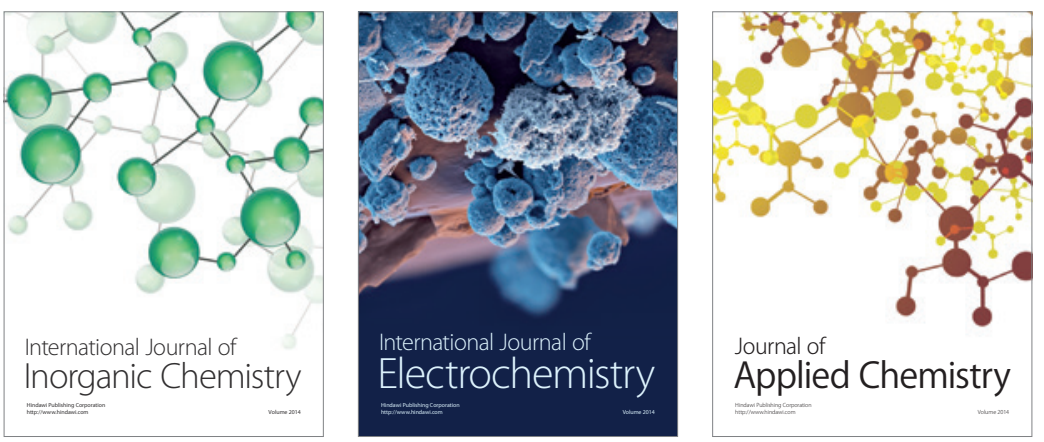

Journal of

Applied Chemistry
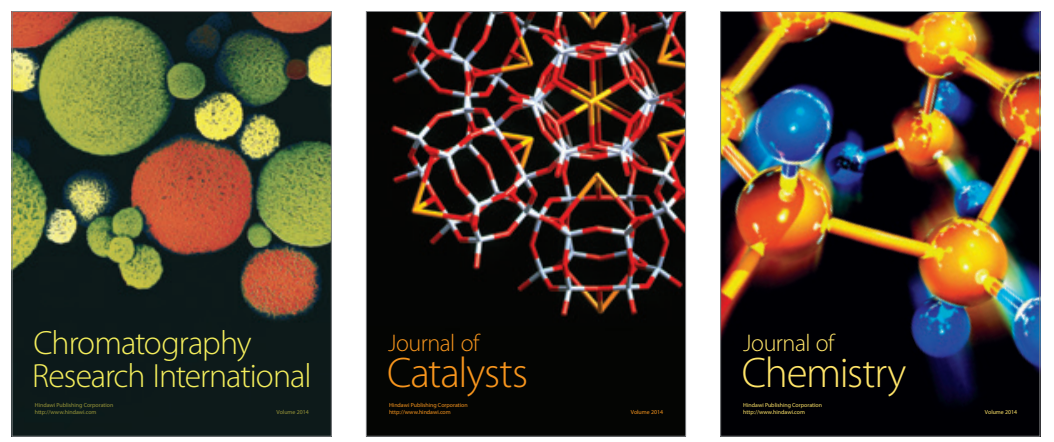
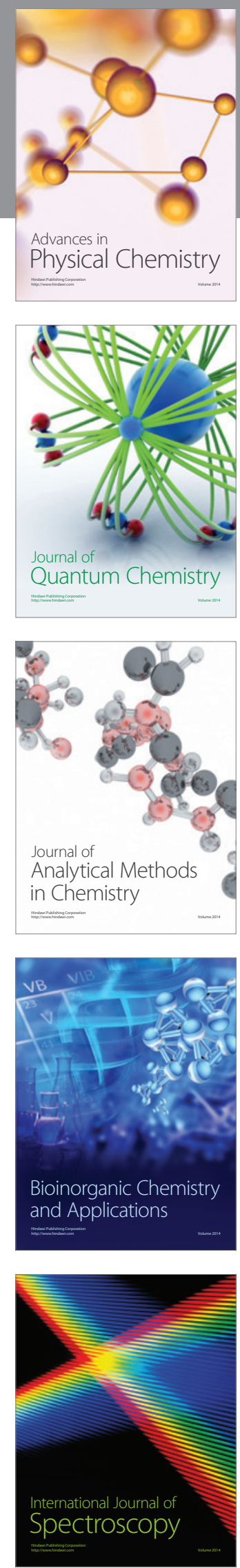